\title{
OPTIMAL PID CONTROLLER DESIGN FOR SPEED CONTROL OF A SEPARATELY EXCITED DC MOTOR: A FIREFLY BASED OPTIMIZATION APPROACH
}

\author{
Pikaso Pal ${ }^{1}$, Rajeeb Dey ${ }^{2}$, Raj Kumar Biswas ${ }^{2}$, Shubhashish Bhakta ${ }^{2}$ \\ 1 Department of Electronics and Instrumentation Engineering, National Institute of \\ Technology Agartala, Tripura-799046, India
}

2 Department of Electrical Engineering, National Institute of Technology Silchar, Assam-788010, India

\begin{abstract}
This paper presents a new approach to determine the optimal proportional-integral-derivative controller parameters for the speed control of a separately excited DC motor using firefly optimization technique. Firefly algorithm is one of the recent evolutionary methods which are inspired by the Firefly's behavior in nature. The firefly optimization technique is successfully implemented using MATLAB software. A comparison is drawn from the results obtained between the linear quadratic regulator and firefly optimization techniques. Simulation results are presented to illustrate the performance and validity of the design method.
\end{abstract}

\section{KEYWORDS}

Separately excited DC motor; Linear Quadratic Regulator; Optimal control; Firefly Algorithm; PID controller Tuning component

\section{INTRODUCTION}

Direct-current (DC) motors find applications in electrical equipments, computer peripherals, robotic manipulators etc due to its excellent speed control characteristics. Therefore the speed control of DC motor attracts the attention of researchers till date as a notable task. For many years, the conventional proportional-integral-derivative (PID) controllers had been used as a control strategy for various industrial processes and motor control applications. Long time and effort is required to tune controller parameters using Ziegler-Nichols frequency response method. Ziegler-Nichols considers system in oscillation mode to realize the tuning procedure, which is not physically realizable [1]. In spite of the simple structure and robustness of PID control strategy, optiming the gains of PID controller is still a difficult task. The Linear quadratic regulator (LQR) method is another method used for this purpose. In LQR performance measure is a quadratic function of state vector and control input. Though the LQR gives a near optimal response, the major drawback of linear quadratic regulator method lies in its lengthy mathematical calculations, 
which is illustrated in this literature. The steps for obtaining the optimized PID gains using the LQR technique are presented in [2]. In this note, an attempt is made to implement the similar procedure for a separately excited dc motor.

Several researchers have implemented heuristic optimization techniques till date. Yadav et al. [3] presented the Genetic algorithm (GA) based PID control for DC motor. GA is inspired by natural selection and evolutionary genetics but degradation is noticed in GA performance when applied to highly epistatic objective functions [4]. Nasri et al. [5] presented the optimal designing of PID control for a linear brushless DC motor using PSO algorithm. Particle swarm optimization (PSO) is a population based search. The major advantages of PSO is its easy implementation and computational efficiency. It is an effective search algorithm which needs to optimise a very few parameters. But major drawback of PSO is its very weak local search ability which results in a fast and premature converge in mid-optimum points [6]. Pal et al. [7] concluded that both the PSO and Firefly algorithm (FFA) performs almost similar in approaching the optimum, provided the process is free of noise. But FFA proves effective and takes less time to reach optimum value in solving non-linear optimization problems and functions in presence of noise. The comparison in performance of FFA with bees algorithm has been investigated in literature [2]. Given a noisy unconstrained mathematical models with continuous design variables the task is to find the optimal solution. A result proves that the efficacy of FFA lies in its unique behavior of attractiveness.

The main objective of this paper is to present an effectiveness of FFA in designing an optimal PID controller for speed control of separately excited DC motor. The remainder of this paper is organized as follows: Section 2 gives a detailed description of the system. Section 3 presents an overview of the optimal tuning rules. MATLAB simulation results are discussed in Section 4. Section 5 finally concludes the paper.

\section{SYSTEM DESCRIPTION}

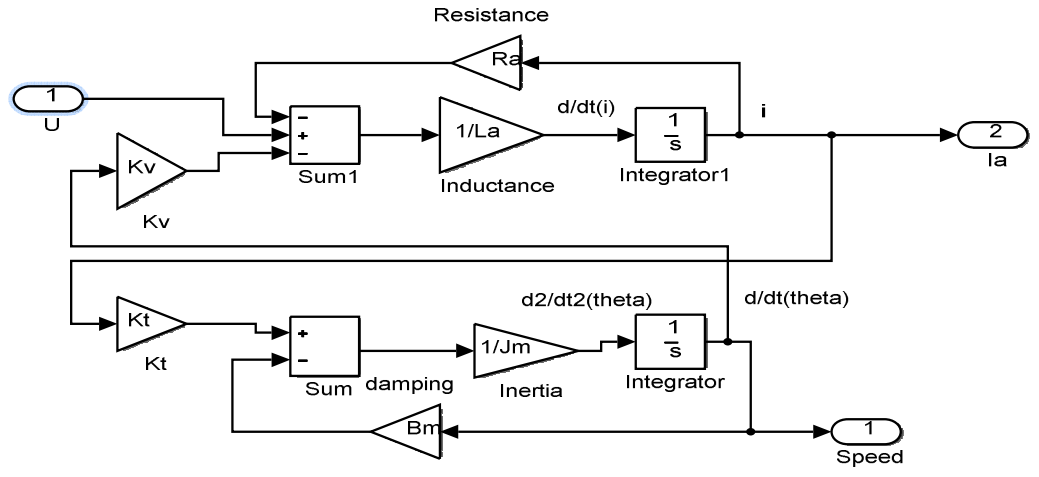

Figure 1. DC motor simulink model. 
Fig.1 shows the DC motor simulink model. The DC motor specification is given in Table 1. By using the electrical equation and the mechanical equation, the armature current and the angular velocity can be measured which describes the DC motor system.

$$
\begin{aligned}
& \frac{d i_{a}}{d t}=\frac{R_{a}}{L_{a}} i_{a}-\frac{K_{v}}{L_{a}} \omega_{a}+\frac{1}{L_{a}} U \\
& \frac{d \omega_{a}}{d t}=\frac{K_{t}}{J_{m}} i_{a}-\frac{B_{m}}{J_{m}} \omega_{a}+\text { external disturbances }
\end{aligned}
$$

where $i_{a}$ is the armature current and $\omega_{a}$ is angular velocity.

In this paper, the external disturbance is considered to be equal to zero. Assuming $i_{a}=X_{1}$ and $\omega_{a}=X_{2}$ and converting these equations into states space form, we get

$$
\frac{d}{d t}\left[\begin{array}{l}
X_{1} \\
X_{2}
\end{array}\right]=\left[\begin{array}{cc}
\frac{R_{a}}{L_{a}} & \frac{-K_{v}}{L_{a}} \\
\frac{K_{t}}{J_{m}} & \frac{-B_{m}}{J_{m}}
\end{array}\right]\left[\begin{array}{l}
X_{1} \\
X_{2}
\end{array}\right]+\left[\begin{array}{c}
\frac{1}{L_{a}} \\
0
\end{array}\right] U+\text { external disturbances }
$$

And the output equation can be written as,

$$
y=\left[\begin{array}{ll}
0 & 1
\end{array}\right]\left[\begin{array}{l}
X_{1} \\
X_{2}
\end{array}\right]+\left[\begin{array}{l}
0 \\
0
\end{array}\right] U
$$

Table1. Separately excited DC motor specification

\begin{tabular}{|c|c|}
\hline DC motor parameter & Value \\
\hline Motor Rating & $3 \mathrm{HP}$ \\
\hline Dc Supply Voltage & $220 \mathrm{~V}$ \\
\hline Motor Rated Current & $4.3 \mathrm{~A}$ \\
\hline Armature Resistance, Ra & $0.6 \Omega$ \\
\hline Armature Inductance,La & $0.008 \mathrm{H}$ \\
\hline Inertia Constant, J & $0.011 \mathrm{Nm}{ }^{\wedge}$ \\
\hline Damping Constant, B & $0.004 \mathrm{Nm} / \mathrm{rad} / \mathrm{sec}$ \\
\hline Torque Constant,Kt & 0.55 \\
\hline Back Emf Constant,Kb & 0.55 \\
\hline Speed & $1800 \mathrm{RPM}$ \\
\hline
\end{tabular}


The matrices $A, B, C$ and $D$ are obtained by considering the specification of separately excited DC motor presented in Table 1 and using equations (3) and (4) as follows

$$
\begin{aligned}
& A=\left[\begin{array}{cc}
-75 & -68.75 \\
50 & -0.3636
\end{array}\right] \quad B=\left[\begin{array}{cc}
125 & 0 \\
0 & -90.9
\end{array}\right] \\
& C=\left[\begin{array}{ll}
0 & 1
\end{array}\right] \quad D=\left[\begin{array}{ll}
0 & 0 \\
0 & 0
\end{array}\right]
\end{aligned}
$$

The system transfer function for the separately excited DC motor is then given by

$$
\frac{Y(s)}{U(s)}=\frac{6250}{s^{2}+75.36 s+3465}
$$

\section{TUNING RULES}

\subsection{LQR based PID Tuning}

This section presents the LQR based tuning of PID parameters for a separately excited dc motor [8]. Fig. 2 shows the LQR based PID tuning of second order system.

$\mathrm{r}(\mathrm{t})$

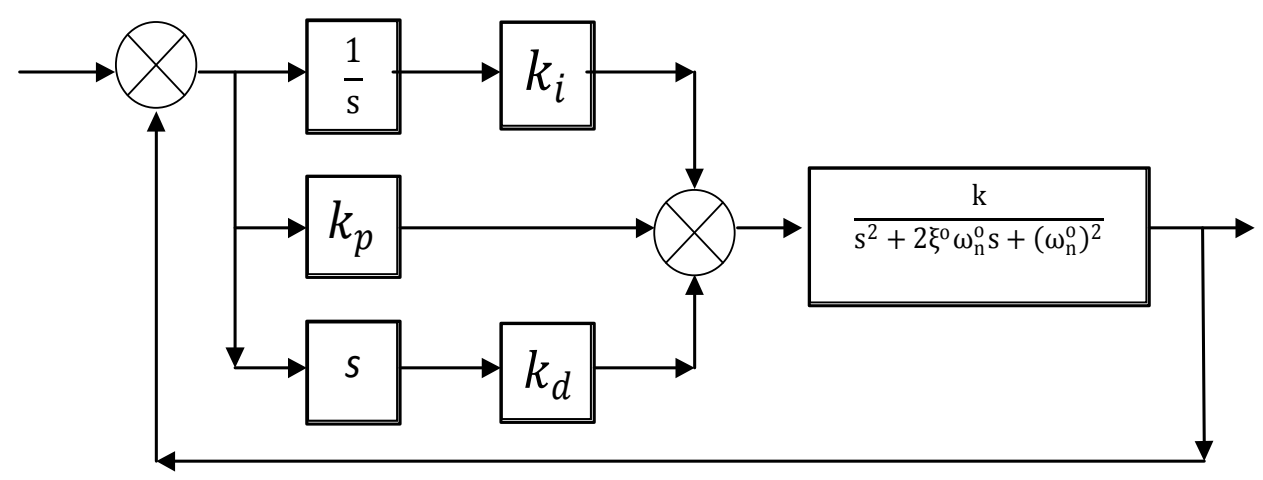

Figure 2. LQR based PID tuning of second order system [8].

The reference input $r(t)$ is considered to be zero as the external set point does not affect the controller design in the state feedback regulator design. With no change in the set point, the relation $y(t)=-e(t)$ is valid for a standard regulator problem. Hence the equation (6) corresponds to

$$
\frac{Y(s)}{U(s)}=\frac{6250}{s^{2}+75.36 s+3465}=\frac{-E(s)}{U(s)}
$$

LQR formulation considers the quadratic cost function given below to be minimized. 


$$
J=\int_{0}^{\infty}\left[X^{T}(t) Q X(t)+u^{T}(t) R u(t)\right] d t
$$

The minimization of above cost function results in an optimal control input given by

$$
u(t)=-R^{-1} B^{T} P X(t)=-F X(\mathrm{t})
$$

where $P$ is a symmetric positive definite solution of continuous algebraic Ricatti equation. The algebraic Ricatti equation is given by,

$$
A^{T} P+P A-P B R^{-1} B^{T} P+Q=0
$$

Equation (7) can be rewritten as

$$
\begin{gathered}
s^{2} E(s)+75.36 s E(s)+3465 E(s)=-6250 U(s) \\
\ddot{e}+75.36 \dot{e}+3465 e=-6250 u
\end{gathered}
$$

Let the state variables be

$$
x_{1}=\int e(t) d t, x_{2}=e(t), x_{3}=\frac{d e(t)}{d t}
$$

Hence equation (12) reduces to

$$
\dot{x}_{3}+75.36 \dot{x}_{2}+3456 x_{2}=-6250 u
$$

Writing equation (14) in states space form

$$
\begin{aligned}
{\left[\begin{array}{c}
\dot{x}_{1} \\
\dot{x}_{2} \\
\dot{x}_{3}
\end{array}\right]=\left[\begin{array}{ccc}
0 & 1 & 0 \\
0 & 0 & 1 \\
0 & -3465 & -75.36
\end{array}\right]\left[\begin{array}{l}
x_{1} \\
x_{2} \\
x_{3}
\end{array}\right]+\left[\begin{array}{c}
0 \\
0 \\
-6250
\end{array}\right] u } \\
F=R^{-1} B^{T} P=R^{-1}\left[\begin{array}{lll}
0 & 0 & -6250
\end{array}\right]\left[\left[\begin{array}{lll}
P_{11} & P_{12} & P_{13} \\
P_{21} & P_{22} & P_{23} \\
P_{31} & P_{32} & P_{33}
\end{array}\right)\right] \\
=-6250 R^{-1}\left[\begin{array}{lll}
P_{13} & P_{23} & P_{33}
\end{array}\right]
\end{aligned}
$$


Weighting matrix $\mathrm{Q}$ is symmetric positive definite and the weighting factor $\mathrm{R}$ is a positive constant. In general, $\mathrm{Q}$ is varied, keeping $\mathrm{R}$ fixed to obtain an optimal control signal from the linear quadratic regulator.

$$
u(t)=-F x(t)=6250 R^{-1}\left[\begin{array}{lll}
P_{13} & P_{23} & P_{33}
\end{array}\right]\left[\begin{array}{l}
x_{1} \\
x_{2} \\
x_{3}
\end{array}\right]
$$

For the closed loop system the characteristic polynomial is given by [8]

$$
s^{3}+\left[2 \xi^{o}+R^{-1} k^{2} P_{33}\right] s^{2}+\left[\left(\omega_{n}^{o}\right)^{2}+R^{-1} k^{2} P_{23}\right] s+R^{-1} k^{2} P_{13}=0
$$

In terms of desired damping ratio and natural frequency, the characteristic polynomial for a closed loop system is given by [8]

$$
s^{3}+(2+m) \xi^{c} \omega_{n}^{c} s^{2}+\left[\left(\omega_{n}^{c}\right)^{2}+2 m\left(\xi^{c}\right)^{2}\left(\omega_{n}^{c}\right)^{2}\right] s+m \xi^{c}\left(\omega_{n}^{c}\right)^{3}=0
$$

Comparing the equations, we get

$$
\begin{aligned}
& k_{i}=\frac{m \xi^{c}\left(\omega_{n}^{c}\right)^{3}}{k} \\
& k_{p}=\frac{\left(\omega_{n}^{c}\right)^{2}+2 m\left(\xi^{c}\right)^{2}-\left(\omega_{n}^{o}\right)^{2}}{k} \\
& k_{d}=\frac{(2+m) \xi^{c}-2 \xi^{o} \omega_{n}^{o}}{k}
\end{aligned}
$$

where, $k_{i}=R^{-1} K P_{13}, k_{p}=R^{-1} K P_{23}$, and $k_{d}=R^{-1} K P_{33}$. The Linear Quadratic regulator (LQR) method considers $m=9, \quad \xi^{c}=1$ and $\omega_{n}^{c}=30$ to determine the optimal values of $k_{p}, k_{i}$ and $k_{d}$.

\subsection{Firefly Algorithm}

The firefly algorithm (FA) is a nature-inspired metaheuristic algorithm. The formulation of this algorithm is inspired by the flashing behaviour of fireflies [7]. In 2007 Xin-She Yang formulated this firefly algorithm assuming

1. All fireflies are unisexual, so the flash of firefly's act as a signal system to attract other fireflies.

2. Brightness of a firefly is proportional to attractiveness and decreases with distance.

3. A given firefly will move randomly if it founds around no other brighter fireflies. 
The attractiveness $\beta$ of a firefly is inversely related to the distance $\mathrm{r}$ and is given by, $\beta=\beta_{o} e^{-\gamma r^{2}}$ where $\beta_{o}$ is the attractiveness at $r=0$. The distance $\mathrm{r}$ or $\mathrm{r}_{\mathrm{ij}}$ between any two fireflies $i$ and $j$ at positions $x_{i}$ and $x_{j}$ respectively is be given by $\mathrm{r}_{\mathrm{ij}}=\left\|x_{i}-x_{j}\right\|$, i.e the difference between the positions of two fireflies. The movement of a firely $i$ with respect to another brighter firefly $j$ is determined by, $x_{i}^{t+1}=x_{i}^{t}+\beta_{o} e^{-\gamma_{i j}^{2}}\left(x_{j}^{t}-x_{i}^{t}\right)+\alpha_{t} \varepsilon_{i}^{t}$ where the first term denotes the present position of the firefly, the second term is due to the attraction and the third term introduces the randomization in the movement. $\alpha_{t}$ is the randomization parameter which can be tuned to vary with the iteration counter $t$ during iterations as given by $\alpha_{t}=\alpha_{o} \delta^{t}$, where $0<\delta<1$, and $\varepsilon_{i}^{t}$ is a vector of random numbers drawn from a gaussian distribution or uniform distribution at time t. The movement of the firefly will become a simple random walk if $\beta_{o}=0 . \gamma=0$ corresponds to no variation and reduces to a variant of particle swarm optimization.

\subsubsection{Implementation of Firefly optimized PID controller}

In PID controller design method, the most common performance criteria are the integral of absolute error (IAE), the integral of time weighted absolute error (ITAE), the integral of squared error (ISE) and the integral of time weighted square error (ITSE). In this paper, the integral of absolute error (IAE) is the fitness function to be minimized.

The IAE criterion is given by $\int_{0}^{\infty}|e| d t$, where e is the difference between the reference speed and the actual speed.

The following section describes the pseudo code [7].

Step1: Initialize the algorithm parameters

Step2: Define the integral absolute error as the objective function.

Step3: Generate an initial population of fireflies $x_{i}(i=1,2, \ldots, n)$

Step4: Determine light intensity for $x_{i}$ by calculating $f\left(x_{i}\right)$

Step5: Define light absorption coefficient $\gamma$

While $t<$ Maximum generation

Make a copy of the generated Firefly

For $i=1: n$ for all $n$ fireflies

For $j=1: n$ for all $\mathrm{n}$ fireflies

If $\left(I_{j}>I_{i}\right)$

Move fireflies $i$ and $j$ according to their attractiveness.

Evaluating new solutions and updating the light intensity for the next iteration

End if

End for $j$

End for $i$

Sorting the fireflies to find the present best

End while

Begin post process on best results obtained. 


\section{RESULTS AND DISCUSSION}

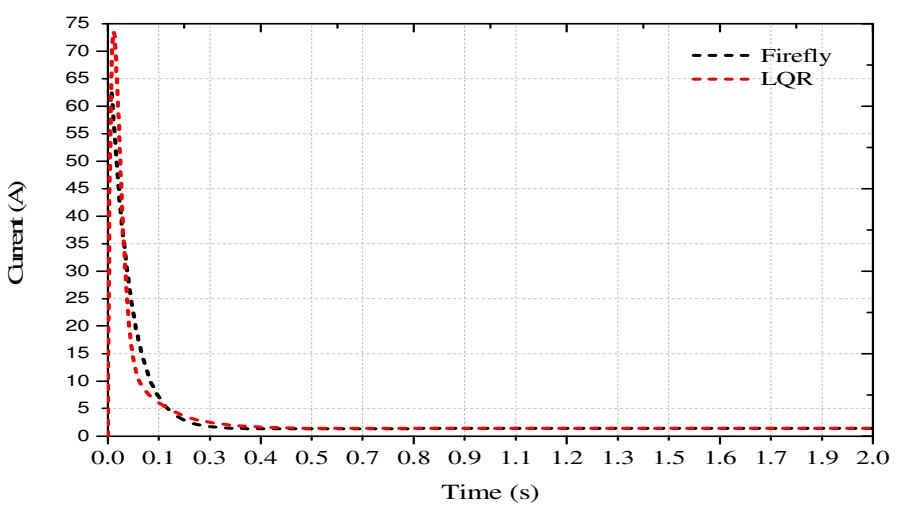

Figure 3. Current response of a separately excited DC motor.

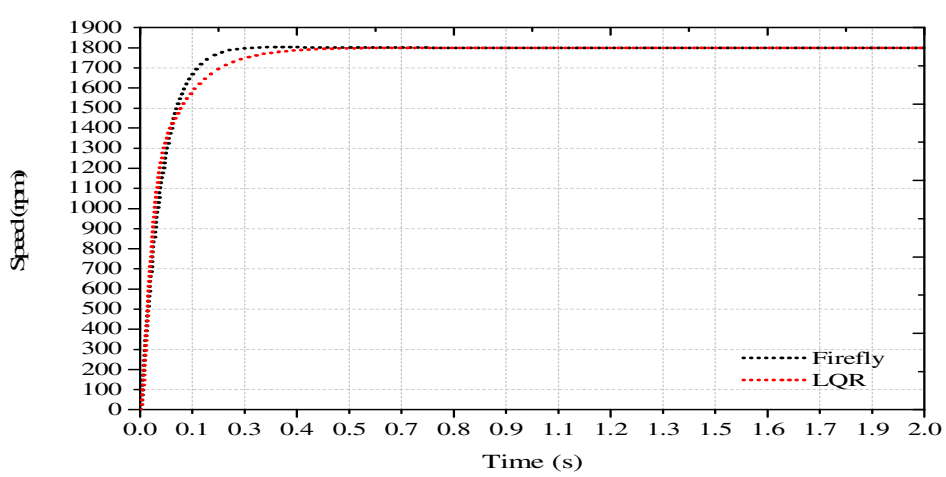

Figure 4. Speed response of a separately excited DC motor.

The optimal value of the gain $k_{p}, k_{i}$ and $k_{d}$ and the time domain response obtained using linear quadratic regulator and firefly algorithm is tabulated below in table 2 and table 3 respectively. The current and speed response of seperataly excited DC motor are shown in Fig. 3 and Fig. 4 repectively. It is comprehended from the study that the FFA provides a better and fast response in terms of time domain specifications like rise time, settling time, peak time as compared to the LQR technique.

Table 2. Optimized PID Parameter

\begin{tabular}{|c|l|l|l|}
\hline & \multicolumn{1}{|c|}{$k_{p}$} & \multicolumn{1}{c|}{$k_{i}$} & \multicolumn{1}{c|}{$k_{d}$} \\
\hline LQR & 2.181604721 & 38.88 & 0.040744652 \\
\hline Firefly & 4.6650 & 44.4648 & 0.2070 \\
\hline
\end{tabular}


Table 3. Time Domain Parameter

\begin{tabular}{|c|c|c|c|c|c|c|}
\hline & Rise Time & $\begin{array}{c}\text { Settling } \\
\text { Time }\end{array}$ & $\begin{array}{c}\text { Peak } \\
\text { Time }\end{array}$ & $\begin{array}{c}\text { Over- } \\
\text { shoot }\end{array}$ & $\begin{array}{c}\text { Under- } \\
\text { shoot }\end{array}$ & $\begin{array}{c}\text { Steady } \\
\text { state } \\
\text { error }\end{array}$ \\
\hline LQR & 0.1412 & 0.2986 & 1.5 & $1.2009 \mathrm{e}-06$ & 0 & 0 \\
\hline Firefly & 0.1233 & 0.2387 & 1.2 & $8.5653 \mathrm{e}-05$ & 0 & 0 \\
\hline
\end{tabular}

The Firefly algorithm determines the optimal PID parameters with an upper bound for $k_{p}, k_{i}$ and $k_{d}$ as 10, 100 and 1 respectively and the lower bound for $k_{p}, k_{i}$ and $k_{d}$ as 0,0 and 0.01 respectively. The parameters for the Firefly algorithm are presented in Table 4.

Table 4. Parameter of Firefly Algorithm

\begin{tabular}{|l|c|}
\hline Number of Fireflies (n) & 5 \\
\hline Maximum Generation & 100 \\
\hline Alpha (randomness) & 0.5 \\
\hline Beta min (initial attractiveness) & 0.2 \\
\hline Gamma (absorption co-efficient) & 0.5 \\
\hline No. of iterations & 500 \\
\hline
\end{tabular}

\section{CONCLUSIONS}

In this paper Firefly optimization technique is implemented to determine the optimal PID controller parameters for a separately excited dc motor. The objective function considered for PID controller design is integral of absolute error. The MATLAB simulation results shows that the proposed algorithm improves the transient performance of the system as compared to the LQR technique with a reduced rise time, settling time, peak time. Moreover, the LQR technique needs time consuming mathematical calculations. In this paper, the external disturbance is set to zero. Our future scope of work will be to carry out the simulation of the above system in presence of external disturbances.

\section{REFERENCES}

[1] Soni, Ritu. Singh, D.B.V. Pandey, Pramod. \& Sharma, Priyanka (2013) "Simulation of optimal speed control for a DC motor using conventional PID controller and Fuzzy logic controller", International Journal of information and computation Technology, Vol. 3, No. 3 , pp 181-188.

[2] Anderson, B.D.O. \& Moore, J.B (1989) "Optimal control: Linear quadratic methods", Prentice -Hall International, Inc., Englewood Cliffs, NJ.

[3] Yadav, S. Misra, V. Omar, S. Dixit, V. Chaubey, P (2014) "Speed control of DC motor using optimal PID Tuning method", National student conference on Advances in Electrical \& Information Communication Technology, pp 94-98. 
[4] He, Jian-Bo., Wang, Qing-Guo., Lee, Tong-Heng.: (2000) "PI/PID controller tuning via LQR approach", Chemical Engineering Science, Vol. 55, No. 13, pp 2429-2439.

[5] Nasri, M. Nezamabadi-pour, H. \& Maghfoori, M (2007) "A PSO based optimum design of PID controller for a linear brushless DC motor", International Journal of Electrical, Robotics, Electronics and Communications Engineering, Vol. 1, No. 2, pp 171-175.

[6] Pinto, A. Elvis, H. Domingues, I. Rocha, L. \& Cruz, S (2010-2011) “The Particle swarm optimization algorithm"

[7] Pal, S.K. Rai, C.S. \& Singh, A.P (2012) "Comparative study of Firefly Algorithm and Particle swarm optimization for noisy non-linear optimization problems", International Journal of intelligent systems and applications, Vol. 10, pp 50-57.

[8] Kumar, V.E., Jerome, J (2013) "LQR based optimal tuning of PID controller for trajectory tracking of magnetic levitation system", International conference on design and manufacturing (Procedia Engineering). Vol. 64,pp 254-264. 